\title{
A Note on Directional Wavelet Transform: Distributional Boundary Values and Analytic Wavefront Sets
}

\author{
Felipe A. Apolonio, ${ }^{1}$ Daniel H. T. Franco, ${ }^{1}$ and Fábio N. Fagundes ${ }^{2}$ \\ ${ }^{1}$ Departamento de Física, Universidade Federal de Viçosa, Avenida Peter Henry Rolfs s/n, \\ Campus Universitário Viçosa, 36570-000 Vioçsa, MG, Brazil \\ ${ }^{2}$ Universidade Federal de Mato Grosso, Campus Sinop, ICNHS, Avenida Alexandre Ferronato 1.200, \\ Distrito Industrial, 78557-267 Sinop, MT, Brazil
}

Correspondence should be addressed to Daniel H. T. Franco, dhtfranco@gmail.com

Received 9 February 2012; Revised 5 April 2012; Accepted 19 April 2012

Academic Editor: Brigitte Forster-Heinlein

Copyright (C 2012 Felipe A. Apolonio et al. This is an open access article distributed under the Creative Commons Attribution License, which permits unrestricted use, distribution, and reproduction in any medium, provided the original work is properly cited.

By using a particular class of directional wavelets (namely, the conical wavelets, which are wavelets strictly supported in a proper convex cone in the $k$-space of frequencies), in this paper, it is shown that a tempered distribution is obtained as a finite sum of boundary values of analytic functions arising from the complexification of the translational parameter of the wavelet transform. Moreover, we show that for a given distribution $f \in S^{\prime}\left(\mathbb{R}^{n}\right)$, the continuous wavelet transform of $f$ with respect to a conical wavelet is defined in such a way that the directional wavelet transform of $f$ yields a function on phase space whose high-frequency singularities are precisely the elements in the analytic wavefront set of $f$.

\section{Introduction}

Wavelets, as well as the theory of distributions, have found applications in various fields of pure and applied mathematics, physics, and engineering. The requirements of modern mathematics, mathematical physics and engineering, have brought the necessity to incorporate ideas from wavelet analysis to the distribution theory, and reciprocally. Over the last decades, a number of authors have studied the relationship between wavelets and the theory of distributions for different purposes (see, e.g., [1-17] and references therein). Particularly related to this note, in $[5,7,11]$, the authors investigate the singularities of tempered distributions via the wavelet transform. In [5], Moritoh introduced a class of wavelet transform as a continuous and microlocal version of the Littlewood-Paley decomposition and compared the wavefront sets defined by his wavelet transform and 
Hörmander's wavefront sets. More recently, Pilipović and Vuletić [11] gave some more precise information concerning the work of Moritoh. They consider a special wavelet transform of Moritoh and gave new definitions of wavefront sets of tempered distributions via that wavelet transform. The major result in [11] is that these wavefront sets are equal to the wavefront sets in the sense of Hörmander in the cases $n=1,2,4,8$. If $n \in \mathbb{N} \backslash\{1,2,4,8\}$, they combined results for dimensions $n=1,2,4,8$ and characterized wavefront sets in $\xi$ directions, where $\xi$ are presented as products of nonzero points of $\mathbb{R}^{n_{1}}, \ldots, \mathbb{R}^{n_{s}}, n_{1}+\cdots+n_{s}=n$, $n_{i} \in\{1,2,4,8\}, i=1, \ldots, s$. In the paper [7], Navarro introduced an analyzing wavelet in $\mathcal{S}\left(\mathbb{R}^{2}\right)$ and an irreducible group action with the property that the associated wavelet transform of a tempered distribution is singular along the wavefront set of the distribution. The main result relates the notion of the wavefront set and the wavelet transform of distributions in $S^{\prime}\left(\mathbb{R}^{2}\right)$. It should be noted that the core of the construction of Navarro (the irreducible group action on $L^{2}\left(\mathbb{R}^{2}\right)$ ) parallels that of Kutyniok and Labate [18], where they consider a different notion of wavefront set based on the concept of continuous shearlet transform.

In this short note, by using a particular class of directional wavelets (namely, the conical wavelets, which are wavelets strictly supported in a proper convex cone in the $k$ space of frequencies $[19,20])$, it is shown that a distribution $f \in S^{\prime}\left(\mathbb{R}^{n}\right)$ is obtained as a finite sum of boundary values of analytic functions arising from the complexification of the variable $x$ corresponding to the location of the continuous wavelet transform of $f$ with respect to directional wavelet $\psi$. Furthermore, we characterize the analytic wavefront set of a tempered distribution in terms of the behavior of its directional wavelet transform. The main results of this work are given in Lemma 3.4 and Theorem 3.5. In Lemma 3.4, we prove that the distributional wavelet transform with respect to the directional wavelet is an analytic function of tempered growth. By using Lemma 3.4, we show that the tempered distributions can be obtained as a finite sum of boundary values of analytic functions of tempered growth (Theorem 3.5). In Section 4, we apply Theorem 3.5 in the study of analytic wavefront set of tempered distributions. We show that, for a given distribution $f \in S^{\prime}\left(\mathbb{R}^{n}\right)$, the wavelet transform of $f$ with respect to a conical wavelet is defined in such a way that the directional wavelet transform of $f$ yields a function on phase space whose high-frequency singularities are precisely the elements in the analytic wavefront set of $f$. In [21], Hörmander introduced the notion of the analytic wavefront set $W F_{a}(f)$ of $f$ as a subset of the cotangent space $T^{*}(X) \backslash 0$, whose projection to $X$ coincides with the analytic singular support of $f$. His definition relies on the use of the Fourier transform of $f$. In this note, following Nishiwada $[22,23]$, we present an alternative definition of $W F_{a}(f)$ in terms of generalized boundary values of analytic functions arising from the complexification of the variable $x$ corresponding to the location of the continuous wavelet transform of $f$ with respect to directional wavelet $\psi$.

\section{A Glance at the Wavelets: Definitions and Basic Properties}

We shall recall in this section some definitions and basic properties of the wavelets. But before we establish some notation. We will use the standard multi-index notation. Let $\mathbb{R}^{n}$ (resp. $\mathbb{C}^{n}$ ) be the real (resp. complex) $n$-space whose generic points are denoted by $x=\left(x_{1}, \ldots, x_{n}\right)$ (resp. $\left.z=\left(z_{1}, \ldots, z_{n}\right)\right)$, such that $x+y=\left(x_{1}+y_{1}, \ldots, x_{n}+y_{n}\right), \lambda x=\left(\lambda x_{1}, \ldots, \lambda x_{n}\right), x \geq 0$ means $x_{1} \geq 0, \ldots, x_{n} \geq 0,\langle x, y\rangle=x_{1} y_{1}+\cdots+x_{n} y_{n}$ and $|x|=\left|x_{1}\right|+\cdots+\left|x_{n}\right|$. Moreover, we define $\alpha=\left(\alpha_{1}, \ldots, \alpha_{n}\right) \in \mathbb{N}_{o}^{n}$, where $\mathbb{N}_{o}$ is the set of nonnegative integers, such that the length 
of $\alpha$ is the corresponding $\ell^{1}$-norm $|\alpha|=\alpha_{1}+\cdots+\alpha_{n}, \alpha+\beta$ denotes $\left(\alpha_{1}+\beta_{1}, \ldots, \alpha_{n}+\beta_{n}\right), \alpha \geq \beta$ means $\left(\alpha_{1} \geq \beta_{1}, \ldots, \alpha_{n} \geq \beta_{n}\right), \alpha !=\alpha_{1} ! \ldots \alpha_{n} !, x^{\alpha}=x_{1}^{\alpha_{1}} \ldots x_{n}^{\alpha_{n}}$, and

$$
D^{\alpha} \varphi(x)=\frac{\partial^{|\alpha|} \varphi\left(x_{1}, \ldots, x_{n}\right)}{\partial x_{1}^{\alpha_{1}} \partial x_{2}^{\alpha_{2}}, \ldots, \partial x_{n}^{\alpha_{n}}} .
$$

We consider two $n$-dimensional spaces- $x$-space and $k$-space-with the Fourier transform defined as follows

$$
\widehat{f}(k)=\mathscr{F}[f(x)](k)=\int_{\mathbb{R}^{n}} d^{n} x f(x) e^{-i\langle k, x\rangle},
$$

while the Fourier inversion formula is

$$
f(x)=\mathcal{F}^{-1}[\widehat{f}(k)](x)=\frac{1}{(2 \pi)^{n}} \int_{\mathbb{R}^{n}} d^{n} k \widehat{f}(k) e^{i\langle k, x\rangle} .
$$

The variable $k$ will always be taken real while $x$ will also be complexified: when it is complex, it will be noted $z=x+i y$.

Definition 2.1. A wavelet is a complex-valued function $\psi$ in $L^{2}\left(\mathbb{R}^{n}, d^{n} x\right)$, satisfying the admissibility condition

$$
C_{\psi}=(2 \pi)^{n} \int \frac{d^{n} k}{|k|^{n}}|\widehat{\psi}(k)|^{2}<\infty
$$

where $\widehat{\psi}$ is the Fourier transform of $\psi$.

If $\psi$ is sufficiently regular-enough to take $\psi \in L^{1}\left(\mathbb{R}^{n}, d^{n} x\right) \cap L^{2}\left(\mathbb{R}^{n}, d^{n} x\right)$ - then the admissibility condition above means that

$$
\widehat{\psi}(0)=0 \Longleftrightarrow \int d^{n} x \psi(x)=0
$$

Definition 2.2. The continuous wavelet transform of a distribution $f(x) \in \mathcal{S}^{\prime}\left(\mathbb{R}^{n}\right)$ with respect to some analyzing wavelet $\psi$ is defined as the following convolution:

$$
\mathbb{W}_{\psi} f\left(x^{\prime}, \ell\right)=\left\langle f(x), \overline{\psi_{x^{\prime}, \ell}(x)}\right\rangle=\int d^{n} x \overline{\psi_{x^{\prime}, \ell}(x)} f(x),
$$

where

$$
\psi_{x^{\prime}, \ell}(x)=\frac{1}{\ell^{n}} \psi\left(\ell^{-1}\left(x-x^{\prime}\right)\right)
$$

with $\ell \in \mathbb{R}_{+}$as the length scale at which we analyze $f(x)$ and $x^{\prime} \in \mathbb{R}^{n}$ as the translation parameter corresponding to the position of the analyzing wavelet $\psi$. 
Remark 2.3. Throughout this article the Fourier transform, the wavelet transform, and boundary values of analytic functions are always interpreted in a distributional sense.

Note that the wavelet transform may also be written as

$$
\mathbb{W}_{\psi} f\left(x^{\prime}, \ell\right)=\frac{1}{(2 \pi)^{n}} \int d^{n} k \widehat{\mathbb{W}_{\psi} f}(k, \ell) e^{i\left\langle k, x^{\prime}\right\rangle},
$$

where

$$
\widehat{\mathbb{W}_{\psi} f}(k, \ell)=\widehat{\psi}(\ell k) \widehat{f}(k),
$$

is the Fourier transform of $\mathbb{W}_{\psi} f$. With this we have the following (see [24, Lemma 8.2.6, page 441] for the one-dimensional space).

Lemma 2.4. For any $f \in L^{2}\left(\mathbb{R}^{n}\right)$, we have

$$
\begin{aligned}
\mathcal{F}\left[\mathbb{W}_{\psi} f\left(x^{\prime}, \ell\right)\right](k, \ell) & =\int_{\mathbb{R}^{n}} d^{n} x \mathbb{W}_{\psi} f\left(x^{\prime}, \ell\right) e^{-i\left\langle k, x^{\prime}\right\rangle} \\
& =\widehat{\psi}(\ell k) \widehat{f}(k) .
\end{aligned}
$$

Remark 2.5. The domain of a wavelet transform is usually the $L^{2}$ space, but the Lemma 2.4 can be extended to $\mathcal{S}^{\prime}\left(\mathbb{R}^{n}\right)$, which is the dual space of $\mathcal{S}\left(\mathbb{R}^{n}\right)$. In particular, see Remark 3.2, the class of conical wavelets belongs to the space $\mathcal{S}\left(\mathbb{R}^{n}\right)$. This allows us to define the directional wavelet transform of a distribution $f \in S^{\prime}\left(\mathbb{R}^{n}\right)$ in such a way that the wavelet transform of $f$ yields a function on phase space whose high-frequency singularities are precisely the elements in the analytic wavefront set of $f$.

\section{Distributional Boundary Values}

In this section, the directional wavelet transform is used to show that analytic functions which satisfy a tempered growth condition obtain distributional boundary values in $S^{\prime}\left(\mathbb{R}^{n}\right)$. Before that, in order to define a directional wavelet, we need some terminology and simple facts concerning cones.

An open set $C \subset \mathbb{R}^{n}$ is called a cone if $C$ (unless specified otherwise, all cones will have their vertices at zero) is invariant under positive homotheties; that is, if for all $\lambda>0$, $\lambda C \subset C$. A cone $C$ is an open connected cone if $C$ is an open connected set. Moreover, $C$ is called convex if $C+C \subset C$ and proper if it contains no any straight line (observe that if $C$ is a cone, then $C$ is proper if and only if $x \in C$ and $x \neq 0$ implie $-x \notin C)$. A cone $C^{\prime}$ is called compact in $C$-we write $C^{\prime} \Subset C$-if the projection $\operatorname{pr} \bar{C}^{\prime} \stackrel{\text { def }}{=} \bar{C}^{\prime} \cap S^{n-1} \subset \operatorname{pr} C \stackrel{\text { def }}{=} C \cap S^{n-1}$, where $S^{n-1}$ is the unit sphere in $\mathbb{R}^{n}$. Being given a cone $C$ in $x$-space, we associate with $C$ a closed convex cone $C^{*}$ in $k$ space which is the set $C^{*}=\left\{k \in \mathbb{R}^{n} \mid\langle k, x\rangle \geq 0, \forall x \in C\right\}$. The cone $C^{*}$ is called the dual cone of $C$.

Definition 3.1. A wavelet $\psi(x)$ is said to be directional if the effective support of its Fourier transform $\widehat{\psi}(k)$ is contained in a convex and proper cone in the $k$-space of frequencies, with 
vertex at the origin, or a finite union of disjoint such cones; in that case, one will usually call $\psi$ multidirectional.

Remark 3.2. According to [19], for us to reach a genuinely directional wavelet $\psi(x)$, it suffices to consider a smooth function $\widehat{\psi}(k)$ with support in the strictly convex cone $C^{*}$ in the $k$ space of frequencies having an arbitrary large number of vanishing moments on the boundary of the supporting cone and behaving inside $C^{*}$ as $P\left(k_{1}, \ldots, k_{n}\right) e^{-\langle k, y\rangle}$, where $y \in C$ and $P(\cdot)$ denotes a polynomial in $n$ variables. In this case, the resulting directional wavelet is also called conical. We also observe that, for all $y \in C$, the exponential $e^{-\langle k, y\rangle}$, considered as a function of $k \in C^{*}$, belongs to the space of rapidly decreasing functions $\mathcal{S}\left(\mathbb{R}^{n}\right)$. Then, $P\left(k_{1}, \ldots, k_{n}\right) e^{-\langle k, y\rangle} \in \mathcal{S}\left(\mathbb{R}^{n}\right)$ too. Since the inverse Fourier transform is a topological isomorphism of $\mathcal{S}\left(\mathbb{R}^{n}\right)$ onto $\mathcal{S}\left(\mathbb{R}^{n}\right)$, it follows that directional wavelet $\psi(x) \in \mathcal{S}\left(\mathbb{R}^{n}\right)$.

In particular, from the above remark, it follows that for a directional wavelet (2.8) can be rewritten as

$$
\mathbb{W}_{\psi} f(z, \ell)=\frac{1}{(2 \pi)^{n}} \int d^{n} k P(\ell k) \widehat{f}(k) e^{i\langle k, z\rangle}
$$

where we have introduced the complex variable $z=x+i \ell y \in \mathbb{R}^{n}+i C$. Remember that here $x$ corresponds to the location of the continuous wavelet transform of $f$ with respect to $\psi$. Consequently, it is clear from (3.1) that the directional wavelet transform, $\mathbb{W}_{\psi} f(x, \ell)$, of a distribution $f \in \mathcal{S}^{\prime}\left(\mathbb{R}^{n}\right)$ is naturally extensible as an analytic function in a domain in $\mathbb{C}^{n}$, for each arbitrary but fixed $\ell>0$. Still, since $C^{*}$ is a regular set $[25$, pages 98, 99], it follows that $\operatorname{supp}\left(\widehat{\mathbb{W}_{\psi} f}\right)=\operatorname{supp}(\widehat{\psi}) \subseteq C^{*}$.

Let $\Omega$ be an open subset in $\mathbb{C}^{n}$. Then we shall denote by $\mathcal{O}(\Omega)$ the space of analytic functions in $\Omega$. Let $C$ be a proper open convex cone, and let $C^{\prime}$ be an arbitrary compact cone contained in $C$. Denote by $T\left(C^{\prime}\right)$ the subset of $\mathbb{C}^{n}$ consisting of all elements whose imaginary parts lie in $C^{\prime} . T\left(C^{\prime}\right)$ is referred to as a tube domain. We will deal with tubes defined as the set of all points $z \in \mathbb{C}^{n}$ such that

$$
T\left(C^{\prime}\right)=\left\{z=x+i y \in \mathbb{C}^{n}\left|x \in \mathbb{R}^{n}, y \in C^{\prime},\right| y \mid<\delta\right\}
$$

where $\delta>0$ is an arbitrary number.

Definition 3.3. Let $Z$ be a complex neighbourhood of $\mathbb{R}^{n}$. An analytic function $f(z) \in \mathcal{O}(Z \cap$ $T\left(C^{\prime}\right)$ ) is said to be of tempered growth if there are an integer $\alpha$ and a constant $\mathbf{M}$ depending on $C^{\prime}$ such that

$$
|f(x+i y)| \leq \mathbf{M}\left(C^{\prime}\right)|y|^{-\alpha}
$$

for all point $z=x+i y$ in $Z \cap T\left(C^{\prime}\right)$.

Lemma 3.4. For an arbitrary but fixed scale $\ell \in \mathbb{R}_{+}$, assume that the function $\mathbb{W}_{\psi} f(z, \ell)$, arising from the complexification of the variable $x$ corresponding to the location of the continuous wavelet transform of $f \in S^{\prime}\left(\mathbb{R}^{n}\right)$ with respect to directional wavelet $\psi$, is analytic in $Z \cap T\left(C^{\prime}\right)$. Then $\mathbb{W}_{\psi} f(z, \ell)$ is of tempered growth as a function of $z$. 
Proof. We start considering the formula

$$
\mathbb{W}_{\psi} f(x+i \ell y, \ell)=\frac{1}{(2 \pi)^{n}} \int_{C^{*}} d^{n} k P(\ell k) \widehat{f}(k) e^{i\langle k, z\rangle}
$$

By Theorem 7.13 of [26], since $P(\ell k)$ is a polynomial and $\widehat{f}(k)$ a tempered distribution, then $P(\ell k) \widehat{f}(k)$ is also tempered. Note that, we can write

$$
P(\ell k)=P_{m}(\ell k)+P_{m-1}(\ell k)+\cdots+P_{0}
$$

where $P_{j}$ is homogeneous of degree $j$ and $P_{m}(\ell k) \neq 0$ when $k \neq 0$. It follows that for some constant $\mathbf{M}$, we have that $|P(\ell k)| \leq \mathbf{M} \ell^{m}|P(k)|$. This implies that $|P(\ell k) \widehat{f}(k)| \leq$ $\mathbf{M} \ell^{m}|P(k) \widehat{f}(k)|$. Still, the character tempered of $P(k) \widehat{f}(k)$ implies that there exist an integer $N$ and a constant $\mathbf{M}_{1}$ such that $P(k) \widehat{f}(k)$ satisfies the estimate

$$
|P(k) \widehat{f}(k)| \leq \mathbf{M}_{1}(1+|k|)^{N} .
$$

Hence,

$$
|P(\ell k) \widehat{f}(k)| \leq \ell^{m} \mathbf{M}_{2}(1+|k|)^{N}
$$

Using the binomial theorem, the above estimate can be rewritten as

$$
|P(\ell k) \widehat{f}(k)| \leq \ell^{m} \mathbf{M}_{2} \sum_{j=0}^{N} c_{j}|k|^{j} .
$$

Now, let $C^{\prime}$ be a cone, such that $C^{\prime} \Subset C$. Then there exists $c>0$ so that $\langle k, \ell y\rangle \geq c|k \| \ell y|$, for all $k \in C^{*}$ and for all $y \in C^{\prime}$. Hence for $x+i \ell y \in \mathbb{R}^{n}+i C^{\prime}$,

$$
\begin{aligned}
\left|\mathbb{W}_{\psi} f(x+i \ell y, \ell)\right| & \leq \frac{\ell^{m}}{(2 \pi)^{n}} \int_{C^{*}} d^{n} k|P(k) \widehat{f}(k)| e^{-\langle k, \ell y\rangle} \\
& \leq \frac{\ell^{m} \mathbf{M}_{2}}{(2 \pi)^{n}} \sum_{j=0}^{N} c_{j} \int_{C^{*}} d^{n} k|k|^{j} e^{-c|k| l y \mid}
\end{aligned}
$$

Following Schwartz [25, Proposition 32, page 39], we get the following:

$$
\begin{aligned}
\left|\mathbb{W}_{\psi} f(x+i \ell y, \ell)\right| & \leq \frac{\ell^{m} \mathbf{M}_{2}}{(2 \pi)^{n}} \sum_{j=0}^{N} c_{j} \sigma^{n-1} \int_{0}^{\infty} d t t^{n+j-1} e^{-c|\ell y| t} \\
& \leq \mathbf{M}_{3}\left(C^{\prime}\right)|\ell y|^{-(n+N)}
\end{aligned}
$$

where $\sigma^{n-1}$ is the area of the unit sphere in $\mathbb{R}^{n}$. 
Now, let $C$ be an open cone of the form $C=\cup_{j=1}^{m} C_{j}, m<\infty$, where each $C_{j}$ is an proper open convex cone. If we write $C^{\prime} \Subset C$, we mean that $C^{\prime}=\cup_{j=1}^{m} C_{j}^{\prime}$ with $C_{j}^{\prime} \Subset C_{j}$. Furthermore, we define by $C_{j}^{*}=\left\{k \in \mathbb{R}^{n} \mid\langle k, x\rangle \geq 0, \forall x \in C_{j}\right\}$ the dual cones of $C_{j}$, such that the dual cones $C_{j}^{*}, j=1, \ldots, m$, have the properties

$$
\begin{gathered}
\mathbb{R}^{n} \backslash\left(\cup_{j=1}^{m} C_{j}^{*}\right), \\
C_{j}^{*} \cap C_{k}^{*}, \quad j \neq k, j, k=1, \ldots, m,
\end{gathered}
$$

that are sets of Lebesgue measure zero. Moreover, assume that $P(\ell k) \widehat{f}(k)$ can be written as $P(\ell k) \widehat{f}(k)=\sum_{j=1}^{m} \lambda_{j}(k) P(\ell k) \widehat{f}(k)$, where $\lambda_{j}(k)$ denotes the characteristic function of $C_{j}^{*}$, $j=1, \ldots, m$. We shall consider the asymptotic property of $\mathbb{W}_{\psi} f(z, \ell)$ as $\ell \rightarrow 0$ for $z=x+i \ell y \in$ $Z \cap T\left(C^{\prime}\right)$.

Theorem 3.5. Let $f \in \mathcal{S}^{\prime}\left(\mathbb{R}^{n}\right)$. Then $f$ can be expressed as a finite sum

$$
f=\sum_{j=1}^{m} b_{C_{j}^{\prime}}\left(\mathbb{W}_{\psi} f_{j}(z, \ell)\right)
$$

where each $\mathbb{W}_{\psi} f_{j}(z, \ell)$, arising from the complexification of the variable $x$ corresponding to the location of the continuous wavelet transform of $f_{j} \in \mathcal{S}^{\prime}\left(\mathbb{R}^{n}\right)$ with respect to directional wavelet $\psi$, is analytic in $\mathrm{Z} \cap T\left(C_{j}^{\prime}\right)$ and of tempered growth, and where $b_{C_{j}^{\prime}}\left(\mathbb{W}_{\psi} f_{j}(z, \ell)\right)$ denotes the boundary value in $S^{\prime}\left(\mathbb{R}^{n}\right)$.

Proof. The proof that each $\mathbb{W}_{\psi} f_{j}(z, \ell)$ is of tempered growth is obtained by the similar way as in Lemma 3.4. Let $\varphi \in \mathcal{S}\left(\mathbb{R}^{n}\right)$. Choose now $y_{0} \in C_{j}^{\prime}$ and write $y=\ell y_{0}$ (this defines a half-line for $0 \neq y_{0} \in C_{j}^{\prime}$ ). Note that with $y=\ell y_{0}$ in (3.1), then $\ell \rightarrow 0$ when $y \rightarrow 0$. Thus, we have

$$
\begin{aligned}
\left\langle b_{C_{j}^{\prime}}\left(\mathbb{W}_{\psi} f_{j}\right), \varphi\right\rangle & =\lim _{y \rightarrow 0} \int_{\mathbb{R}^{n}} d^{n} x \mathbb{W}_{\psi} f_{j}(x+i y, \ell) \varphi(x) \\
& =\lim _{y \rightarrow 0} \int_{\mathbb{R}^{n}} d^{n} x\left(\frac{1}{(2 \pi)^{n}} \int_{C_{j}^{*}} d^{n} k \lambda_{j}(k) P(\ell k) \widehat{f}(k) e^{i\langle k, x+i y\rangle}\right) \varphi(x) \\
& =\lim _{y \rightarrow 0} \frac{1}{(2 \pi)^{n}} \int_{C_{j}^{*}} d^{n} k \lambda_{j}(k) P(\ell k) \widehat{f}(k) \widehat{\varphi}(-k) e^{-\langle k, y\rangle} \\
& =\frac{1}{(2 \pi)^{n}} \int_{C_{j}^{*}} d^{n} k \lambda_{j}(k) \widehat{f}(k) \widehat{\varphi}(-k) \\
& =\left\langle f_{j}, \varphi\right\rangle .
\end{aligned}
$$


Hence, using the linearity of $f \in \mathcal{S}^{\prime}\left(\mathbb{R}^{n}\right)$ and the assumptions (3.11), we obtain that

$$
\langle f, \varphi\rangle=\sum_{j=1}^{m}\left\langle f_{j}, \varphi\right\rangle=\sum_{j=1}^{m}\left\langle b_{C_{j}^{\prime}}\left(\mathbb{W}_{\psi} f_{j}\right), \varphi\right\rangle
$$

Thus, the limit of each $\mathbb{W}_{\psi} f_{j}(z, \ell)$ as $C_{j}^{\prime} \ni y \rightarrow 0$ exists in $\mathcal{S}^{\prime}\left(\mathbb{R}^{n}\right)$; that is, $f$ admits the distributional boundary value $\sum_{j=1}^{m} b_{C_{j}^{\prime}}\left(\mathbb{W}_{\psi} f_{j}\right)$ in the sense of weak convergence. But from [27, Corollary 1, page 358], the latter implies strong convergence since $\mathcal{S}\left(\mathbb{R}^{n}\right)$ is Montel.

\section{Analytic Wavefront Set}

From what we have seen in the previous section, a distribution $f \in S^{\prime}\left(\mathbb{R}^{n}\right)$ is obtained as a finite sum of boundary values of analytic functions $\mathbb{W}_{\psi} f_{j}(z, \ell)$, with $j=(1, \ldots, m)$, arising from the complexification of the variable $x$ corresponding to the location of the continuous wavelet transform of $f_{j}$ with respect to directional wavelet $\psi$, and where a tempered growth condition was described to characterize such boundary values. We now translate growth condition in terms of the analytic wavefront set.

Definition 4.1. Let $f \in \mathcal{S}^{\prime}\left(\mathbb{R}^{n}\right)$, such that $f=b_{C}\left(\mathbb{W}_{\psi} f(z, \ell)\right)$, where $b_{C}\left(\mathbb{W}_{\psi} f(z, \ell)\right)$ denotes the strong boundary value in $S^{\prime}\left(\mathbb{R}^{n}\right)$ of an analytic function $\mathbb{W}_{\psi} f(z, \ell)$ arising from the complexification of the variable $x$ corresponding to the location of the continuous wavelet transform of $f$ with respect to directional wavelet $\psi$. Let $q=\left(x_{0}, k_{0}\right)$. Then, $q \notin W F_{a}(f)$ if and only if there exist $\mathbf{M}\left(C^{\prime}\right)$ and $N$ for which we have the estimate

$$
\left|\mathbb{W}_{\psi} f(z, \ell)\right| \leq \mathbf{M}\left(C^{\prime}\right)|\ell y|^{-N}, \quad z=x+i \ell y \in Z \cap T\left(C^{\prime}\right)
$$

$W F_{a}(f)$ is called analytic wavefront set of $f$.

Proposition 4.2. Let $f \in S^{\prime}\left(\mathbb{R}^{n}\right)$ be the boundary value, in the distributional sense, of a function $\mathbb{W}_{\psi} f(z, \ell)$ analytic in $Z \cap T\left(C^{\prime}\right)$, arising from the complexification of the variable $x$ corresponding to the location of the continuous wavelet transform of $f$ with respect to directional wavelet $\psi$ and which satisfies the estimate (3.3). Then near $x_{0}$ the fibre $\left.W F_{a}(f)\right|_{x_{0}}$ is contained in $C^{*}$.

Proof. Let $\left.W F_{a}(f)\right|_{x_{0}}=\left\{k \in\left(\mathbb{R}^{n} \backslash 0\right) \mid\left(x_{0}, k\right) \in W F_{a}(f)\right\}$ be the fibre over $x_{0}$. Let $\left\{C_{j}^{*}\right\}_{j \in L}$ be a finite covering of closed properly convex cones of $C^{*}$. Decompose $P(\ell k) \widehat{f}(k)$ as follows:

$$
P(\ell k) \widehat{f}(k)=\sum_{j=1}^{m} \lambda_{j}(k) P(\ell k) \widehat{f}(k)
$$

where $\lambda_{j}(k)$ denotes the characteristic function of $C_{j}^{*}, j \in L$. Then, by Theorem 3.5, the decomposition (4.2) will induce a representation of $f$ in the form of a sum of boundary values of functions $\mathbb{W}_{\psi} f_{j}(z, \ell)$, such that $\mathbb{W}_{\psi} f_{j}(z, \ell) \rightarrow f_{j}$ in the strong topology of $\mathcal{S}^{\prime}\left(\mathbb{R}^{n}\right)$ as 
$y \rightarrow 0, y \in C_{j}^{\prime} \Subset C_{j}$. According to Lemma 3.4, the family of functions $\mathbb{W}_{\psi} f_{j}(z, \ell)$ satisfies the following estimate:

$$
\left|\mathbb{W}_{\psi} f_{j}(z, \ell)\right| \leq \mathbf{M}\left(C^{\prime}\right)|\ell y|^{-N}, \quad z=x+i \ell y \in Z \cap T\left(C^{\prime}\right),
$$

unless $\langle k, Y\rangle<0$ for $k \in C_{j}^{*}$ and $Y \in-C_{j}^{\prime}$, with $|Y|<\delta$. Then, the cones of "bad" directions responsible for the singularities of these boundary values are contained in the dual cones of the base cones. So we have the inclusion

$$
W F_{a}(f) \subset \mathbb{R}^{n} \times\left(\cup_{j} C_{j}^{*}\right)
$$

Then, by making a refinement of the covering and shrinking it to $C^{*}$, we obtain the desired result.

Remark 4.3. It is remarked that in [21] the fiber over $x_{0},\left.W F_{a}(f)\right|_{x_{0}}$, is completely characterized by sequences of type $f_{N}=\phi_{N} f$, where $\left\{\phi_{N}\right\}$ is a bounded sequence in $C_{0}^{\infty}(X)$ which is equal to 1 in a common neighborhood of $x_{0}$ and satisfies the following estimate:

$$
\left|D^{\alpha+\beta} \phi_{N}\right| \leq C_{\alpha}(C N)^{|\beta|}, \quad \text { if }|\beta| \leq N
$$

For the existence of such functions, we refer to Lemma 2.2 in [21].

We can meet Definition 4.1 and Proposition 4.2 in the following proposition.

Proposition 4.4. Let $f \in \mathcal{S}^{\prime}\left(\mathbb{R}^{n}\right)$ and $\left(x_{0}, k_{0}\right) \in T^{*}\left(\mathbb{R}^{n}\right) \backslash 0$, where $T^{*}\left(\mathbb{R}^{n}\right) \backslash 0:=\mathbb{R}^{n} \times\left(\mathbb{R}^{n} \backslash 0\right)$. Then $\left(x_{0}, k_{0}\right) \notin W F_{a}(f)$ if and only if there exists a finite family $\left\{C_{j}\right\}$ of proper open convex cones in $\mathbb{R}^{n}$, a complex neighborhood $Z$ of $x_{0}$ in $\mathbb{C}^{n}$ and a decomposition of $f$

$$
f=\sum_{j=1}^{m} b_{C_{j}^{\prime}}\left(\mathbb{W}_{\psi} f_{j}(z, \ell)\right)
$$

with each $\mathbb{W}_{\psi} f_{j}(z, \ell) \in \mathcal{O}\left(Z \cap T\left(C_{j}^{\prime}\right)\right)$ being of tempered growth and analytic near to $x_{0}$ for every $j$ satisfying $C_{j}^{\prime} \subset\left\{y \mid\left\langle k_{0}, y\right\rangle \geq 0\right\}$, and where $b_{C_{j}^{\prime}}\left(\mathbb{W}_{\psi} f_{j}(z, \ell)\right)$ denotes the boundary value, in the distributional sense, of analytic functions $\mathbb{W}_{\psi} f_{j}(z, \ell)$ arising from the complexification of the variable $x$ corresponding to the location of the continuous wavelet transform of $f$ with respect to directional wavelet $\psi$.

Note that the above proposition shows that a decomposition of a tempered distribution $f$ into a sum of boundary values of analytic functions is equivalent to a decomposition of analytic wavefront set of $f$ since the fibre $\left.W F_{a}(f)\right|_{x_{0}}$ is contained in $\cup_{j} C_{j}^{*}$. Moreover, the decomposition (4.6) is carried out in the space of $C^{\infty}$ functions, provided that $f$ is $C^{\infty}$.

Finally, we recall that in [28] Hörmander defined the wavefront set, $W F(f)$, for a distribution as the set of points in the cotangent space which must be characteristic for every pseudodifferential operator $P$ such that $P f \in C^{\infty}$. It is clear that $W F(f) \subset W F_{a}(f)$. 
Following Nishiwada [22] another characterization of $W F(f)$ can be obtained based on the above results.

Proposition 4.5. Let $U$ be an open set in $\mathbb{R}^{n},\left(x_{0}, k_{0}\right) \in T^{*}(U) \backslash 0$ and $f \in \mathcal{S}^{\prime}(U)$. Then $\left(x_{0}, k_{0}\right) \notin$ $W F(f)$ if there exists a finite family $\left\{C_{j}\right\}$ of proper open convex cones in $\mathbb{R}^{n}$, with $j=1, \ldots, m, a$ complex neighborhood $\mathrm{Z}$ of $x_{0}$ and a decomposition of $f$ near $x_{0}$

$$
f=\sum_{j=1}^{m} b_{C_{j}^{\prime}}\left(\mathbb{W}_{\psi} f_{j}(z, \ell)\right) \text { in } U,
$$

with $\mathbb{W}_{\psi} f_{j}(z, \ell) \in \mathcal{O}\left(Z \cap T\left(C_{j}^{\prime}\right)\right)$ being of tempered growth, such that $b_{C_{j}^{\prime}}\left(\mathbb{W}_{\psi} f_{j}(z, \ell)\right) \in C^{\infty}$ near $x_{0}$ for every $j$ with $C_{j}^{\prime} \subset\{y \mid\langle k, \ell y\rangle \geq 0\}$.

Proof. The proof is similar to the proof of the first part of Theorem 3.4 in [23].

\section{Acknowledgment}

The authors are specially indebted to the referees for valuable comments, suggestions, and constructive criticisms, which improved the presentation of the results. F. A. Apolonio and F. N. Fagundes are supported by the Coordenação de Aperfeiçoamento de Pessoal de Nível Superior (CAPES) agency.

\section{References}

[1] G. G. Walter, "Wavelets and generalized functions," in Wavelets: A Tutorial in Theory and Applications, C. K. Chui, Ed., vol. 2, pp. 51-70, Academic Press, 1992.

[2] M. Holschneider, "Localization properties of wavelet transforms," Journal of Mathematical Physics, vol. 34, no. 7, pp. 3227-3244, 1993.

[3] M. Holschneider, Wavelets: An Analysis Tool, Oxford University Press, 1995.

[4] J. Schmeelk and A. Takači, "Wavelet transforms in generalized Fock spaces," International Journal of Mathematics and Mathematical Sciences, vol. 20, no. 4, pp. 657-672, 1997.

[5] S. Moritoh, "Wavelet transforms in euclidean spaces-their relation with wave front sets and besov, Triebel-Lizorkin spaces," The Tohoku Mathematical Journal, vol. 47, no. 4, pp. 555-565, 1995.

[6] M. Holschneider, "Some directional microlocal classes defined using wavelet transforms," in Comptes Rendues des Ateliers sur les Fonctions Splines et la Theorie des Ondelettes, S. Dubuc, G. Deslauries, J.-M. Lina, and B. MacGibbon, Eds., vol. 18, pp. 115-133, 1999.

[7] J. Navarro, "Singularities of distributions via the wavelet transform," SIAM Journal on Mathematical Analysis, vol. 30, no. 2, pp. 454-467, 1999.

[8] B. K. Sohn and D. H. Pahk, "Analytic representation of generalized tempered distributions by wavelets," Research Institute for Mathematical Sciences, vol. 37, no. 2, pp. 141-149, 2001.

[9] R.-Q. Jia, J. Wang, and D.-X. Zhou, "Compactly supported wavelet bases for Sobolev spaces," Applied and Computational Harmonic Analysis, vol. 15, no. 3, pp. 224-241, 2003.

[10] R. S. Pathak, "The wavelet transform of distributions," The Tohoku Mathematical Journal, vol. 56, no. 3, pp. 411-421, 2004.

[11] S. Pilipović and M. Vuletić, "Characterization of wave front sets by wavelet transforms," The Tohoku Mathematical Journal, vol. 58, no. 3, pp. 369-391, 2006.

[12] S. Pilipović and N. Teofanov, "Multiresolution expansion, approximation order and quasiasymptotic behavior of tempered distributions," Journal of Mathematical Analysis and Applications, vol. 331, no. 1, pp. 455-471, 2007.

[13] K. Saneva, "Application of the quasiasymptotic boundedness of distributions on wavelet transform," Institut Mathématique, vol. 86, no. 100, pp. 115-122, 2009. 
[14] K. Saneva and J. Vindas, "Wavelet expansions and asymptotic behavior of distributions," Journal of Mathematical Analysis and Applications, vol. 370, no. 2, pp. 543-554, 2010.

[15] J. Vindas, S. Pilipović, and D. Rakić, "Tauberian theorems for the wavelet transform," The Journal of Fourier Analysis and Applications, vol. 17, no. 1, pp. 65-95, 2011.

[16] S. Pilipović and J. Vindas, "Multidimensional Tauberian theorems for wavelet and non-wavelet transforms," http:/ /arxiv.org/abs/1012.5090v2.

[17] R. Ashurov and A. Butaev, "On continuous wavelet transforms of distributions," Applied Mathematics Letters, vol. 24, no. 9, pp. 1578-1583, 2011.

[18] G. Kutyniok and D. Labate, "Resolution of the wavefront set using continuous shearlets," Transactions of the American Mathematical Society, vol. 361, no. 5, pp. 2719-2754, 2009.

[19] J.-P. Antoine, R. Murenzi, and P. Vandergheynst, "Directional wavelets revisited: cauchy wavelets and symmetry detection in patterns," Applied and Computational Harmonic Analysis, vol. 6, no. 3, pp. 314-345, 1999.

[20] J.-P. Antoine, R. Murenzi, P. Vandergheynst, and S. T. Ali, Two-Dimensional Wavelets and Their Relatives, Cambridge University Press, 2004.

[21] L. Hörmander, "Uniqueness theorems and wave front sets for solutions of linear differential equations with analytic coefficients," Communications on Pure and Applied Mathematics, vol. 24, pp. 671-704, 1971.

[22] K. Nishiwada, "On the local surjectivity of analytic partial differential operators in the space of distributions with given wave front sets," Surikaiseki-kenkyusho kokyuroku, Research Institute for Mathematical Sciences, vol. 239, p. 19, 1975.

[23] K. Nishiwada, "On local characterization of wave front sets in terms of boundary values of holomorphic functions," Research Institute for Mathematical Sciences, vol. 14, no. 2, pp. 309-320, 1978.

[24] L. Debnath and P. Mikusiński, Introduction to Hilbert Spaces, Elsevier, 3rd edition, 2005.

[25] L. Schwartz, Méthodes Mathématiques pour les Sciences Physiques, Hermann, 2nd edition, 1979.

[26] W. Rudin, Functional Analysis, McGraw-Hill Book, 1973.

[27] F. Trèves, Topological Vector Spaces, Distributions and Kernels, Academic Press, 1967.

[28] L. Hörmander, "Fourier integral operators I," Acta Mathematica, vol. 127, no. 1-2, pp. 79-183, 1971. 


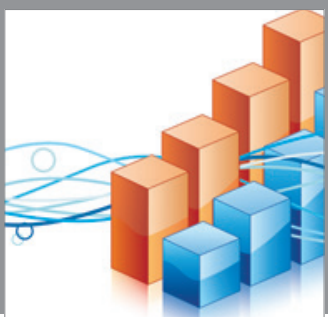

Advances in

Operations Research

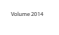

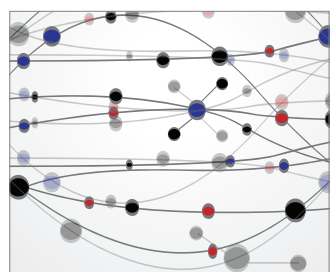

\section{The Scientific} World Journal
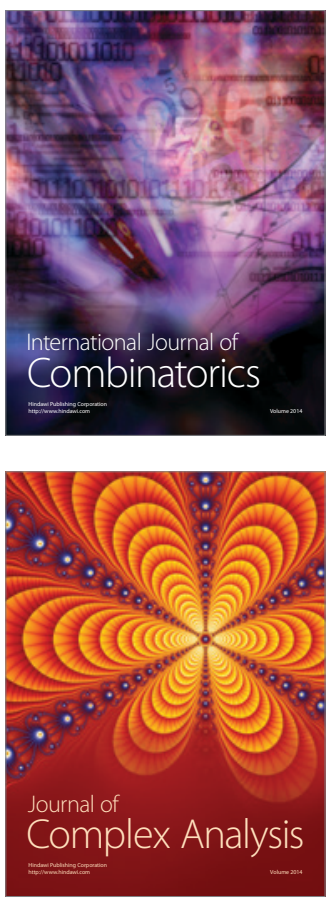

International Journal of

Mathematics and

Mathematical

Sciences
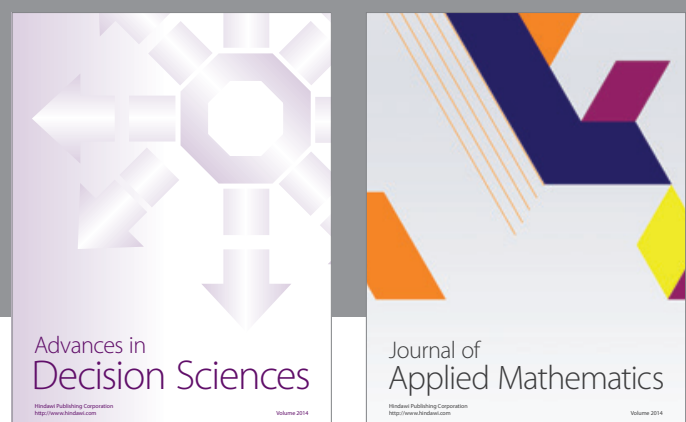

Journal of

Applied Mathematics
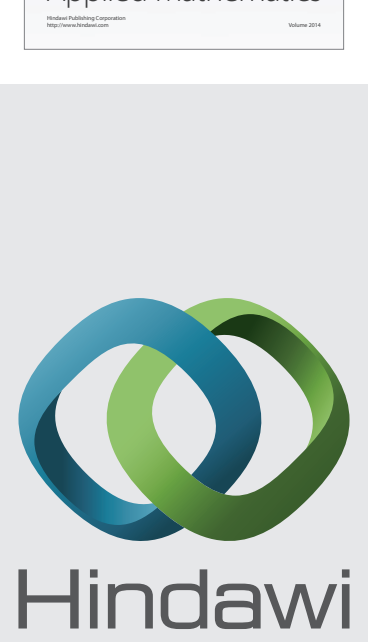

Submit your manuscripts at http://www.hindawi.com
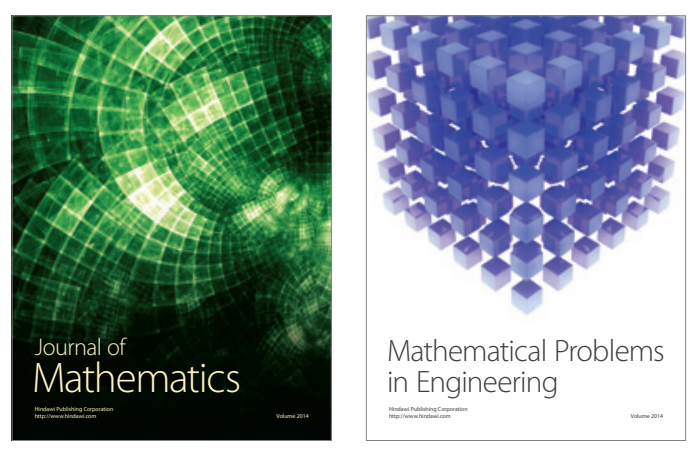

Mathematical Problems in Engineering
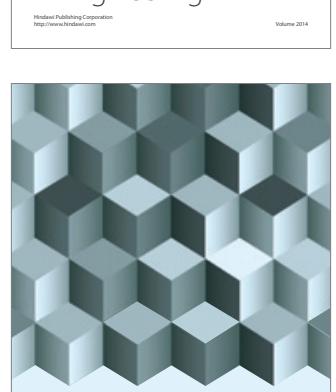

Journal of

Function Spaces
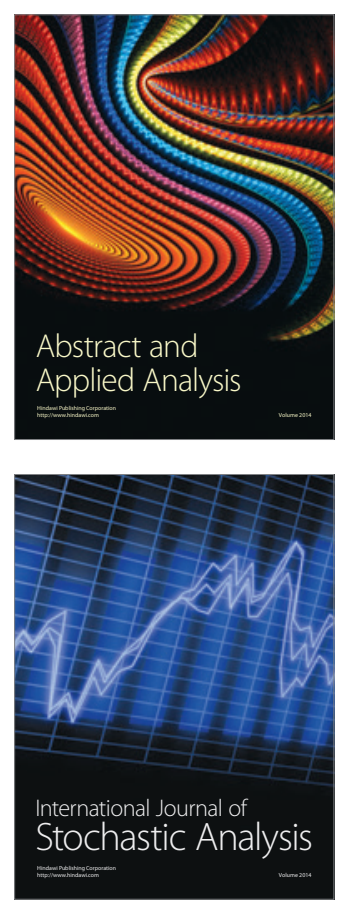

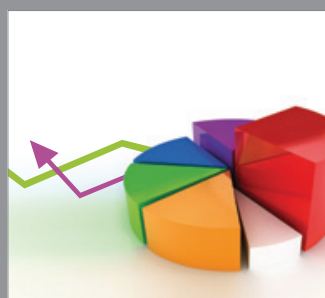

ournal of

Probability and Statistics

Promensencen
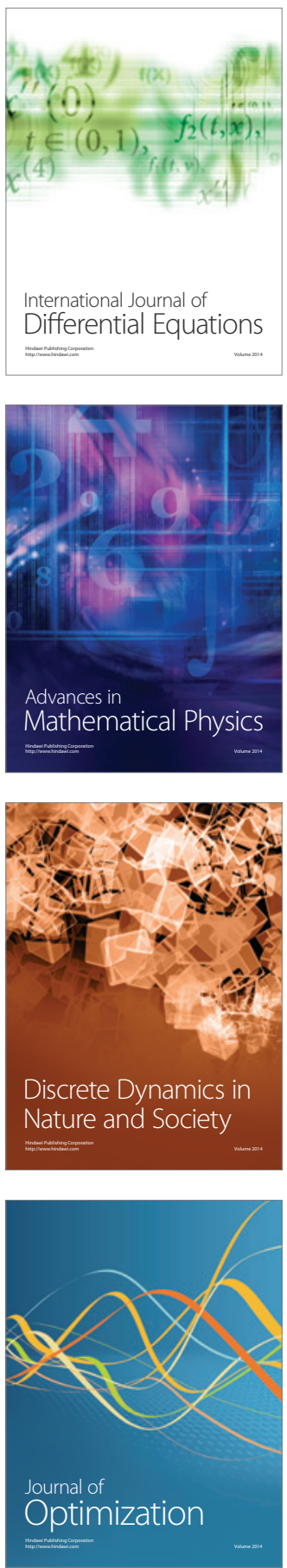